\title{
Topical use of indomethacin on the day of cataract surgery
}

\author{
A E T Searle, J L Pearce, D E Shaw
}

\begin{abstract}
The use of topical indomethacin in the prevention of surgically induced miosis has been documented. ${ }^{2}$ However, in these previous prospective trials this prostaglandin synthetase inhibitor was administered the day before surgery. With the frequency of 'day case' cataract surgery increasing, an efficient preoperative mydriatic regimen is important. In this study we considered the use of topical indomethacin as an addition to a regimen already implemented. One hundred and fourteen eyes underwent intercapsular cataract surgery, of which 64 were randomised to receive topical aqueous indomethacin one hour beforehand, and 50 eyes, which did not receive indomethacin formed the comparison group. Topical indomethacin reduced the miosis which occurs during cataract surgery whether performed under local or general anaesthesia. The operating time was shorter for eyes with less surgically induced miosis.
\end{abstract}

Mydriasis achieved preoperatively, before interor endocapsular cataract surgery, may influence the length of each surgical procedure and the complications encountered. Topical indomethacin has been shown to maintain pupil dilatation during cataract surgery, ${ }^{12}$ but in each study this prostaglandin synthetase inhibitor was administered on the evening before surgery. It is not always practicable to administer eye drops on the day before surgery, and mydriatic regimens may start only 1-2 hours preoperatively. The desired effect of indomethacin in such circumstances is unproved.

In this study the effect on perioperative pupil size of administering topical indomethacin one hour before cataract surgery was investigated.

\section{Patients and methods}

Ninety two patients undergoing intercapsular cataract surgery by one surgeon (JLP), at the Alexandra Hospital, Redditch, between 2 November 1987 and March 1988 were included: 22 patients underwent bilateral surgery and 70 unilateral surgery. The patients' ages ranged from 14 to 95 years (mean 71 16 years). A total of 114 eyes were observed.

All patients falling into one of the following groups were excluded: diabetes mellitus; receiving systemic drugs with autonomic effects; past history of glaucoma, uveitis, or herpes zoster ophthalmicus; previous intraocular surgery; previous history of trauma to the eyes, face, or head; and abnormal pupillary responses to light stimulation.
Patients were randomly allocated to a group receiving preoperative topical indomethacin that is, to a treatment group or to a nontreatment group - by the first author. In cases of bilateral surgery both eyes were allocated to the same group.

\section{MYDRIATIC REGIMEN}

One hour preoperatively all eyes received the following topical mydriatic treatment, administered by the nursing staff on the ward: 1 drop of cyclopentolate $1 \%$ and phenylephrine $10 \%$ every 15 minutes for $\mathbf{4 5}$ minutes before surgery.

Eyes allocated to the treatment group also received: 1 drop of aqueous indomethacin $1 \%$ (supplied by Merck Sharp and Dohme on a named patient basis) every 15 minutes for 45 minutes before surgery. Permission to use this drug was obtained from the local ethical committee.

\section{ANAESTHESIA}

Thirty eyes underwent cataract surgery with local anaesthesia (LA alone). Eighty four eyes underwent cataract surgery with a combination of local and general anaesthesia (LA plus GA). All patients undergoing LA alone received one hour before surgery a peribulbar injection of $4 \mathrm{ml}$ of a 50:50 mixture of $4 \%$ plain lignocaine and $0.75 \%$ bupivacaine to attain ocular anaesthesia and akinaesia and $4 \mathrm{ml}$ of the same mixture as a facial injection by Van Lindt's method to attain orbicularis oculi akinaesia.

All patients undergoing LA plus GA received a peribulbar injection of $0.75 \%$ bupivacaine at the induction of general anaesthesia within five minutes of surgery.

\section{CATARACT SURGERY}

Each intercapsular cataract extraction was performed by JLP. Information on the allocation of each eye to a treatment or non-treatment group was not made available to the surgeon preoperatively.

The operating technique, which did not alter during the four-month period, included: $(a)$ measurement of the horizontal diameter of the pupil to $0.5 \mathrm{~mm}$ with a caliper under view with the operating microscope, after insertion of the superior rectus suture, by the surgeon; $(b)$ a corneoscleral wound; $(c)$ anterior capsulotomy after injection of sodium hyaluronate; $(d)$ an intercapsular technique of irrigation and aspiration of cortical lens matter; $(e)$ insertion of an intraocular posterior chamber lens into the capsular bag and positioning with a Sinskey hook; $(f)$ anterior capsulectomy under sodium hyaluronate.

During the cataract surgery a balanced salt solution containing $0.3 \mathrm{ml}$ of $1: 1000$ adrenaline 
in $250 \mathrm{ml}$ was used to wet the cornea and as an intraocular infusion.

All eyes developing interoperative complications were excluded.

OPERATING TIME

The time elapsing between both pupil measurements was recorded to the nearest second with a stop clock in the theatre.

Sixty-four eyes were included in the treatment group, of which 50 underwent cataract surgery with combined LA plus GA and 14 underwent cataract surgery with LA alone. Fifty eyes were included in the non-treatment group, of which 34 eyes underwent cataract surgery with combined LA plus GA and 16 eyes underwent cataract surgery with LA alone. Comparison of the initial and continual mydriatic effect was made between the treatment and non-treatment groups of eyes, and means and standard deviations are given. As common statistical methods cannot be used when the outcome variable is not independent, and eyes within a person are unlikely to be independent, the method of Rosner ${ }^{3}$ was employed in which a correction for this intrasubject correlation is made. One patient treated on one side only, aged 14 years, the youngest, was excluded from the analysis, as his operation time (35 minutes) was 10 SDs away from the mean. The youngest subject in the study after completion of the study was 27 years old.

\section{Results}

The mean preoperative pupil diameter of all 113 studied eyes was $8.2 \mathrm{~mm}(\mathrm{SD} 1 \cdot 1)$, and the mean reduction after surgery was $1.5 \mathrm{~mm}(\mathrm{SD} 1.0)$ (the proportionate change being $0 \cdot 19(\mathrm{SD} 0 \cdot 12))$. The mean operation time was $15.6 \mathrm{~min}(\mathrm{SD} 1.9)$.

The preoperative pupil diameters were inversely related to the age of the patient, this relationship being only marginally significant $(\mathrm{p}=0.1)$ but were larger in the $\mathrm{LA}+\mathrm{GA}$ group $(8 \cdot 3$, SD 0.9$)$ than in the LA alone group $(7 \cdot 8$, SD $1 \cdot 3, p=0 \cdot 044)$. The initial pupil diameters were not significantly different between the treatment and non-treatment groups.

The change in pupil diameter during surgery was inversely related to the duration of surgery $(\mathrm{p}<0.001)$ (Fig 1) and to the treatment group $(\mathrm{p}<0.001)$ (Table I). These effects were inde-

Figure 1: Change in pupil diameter by length of operation.

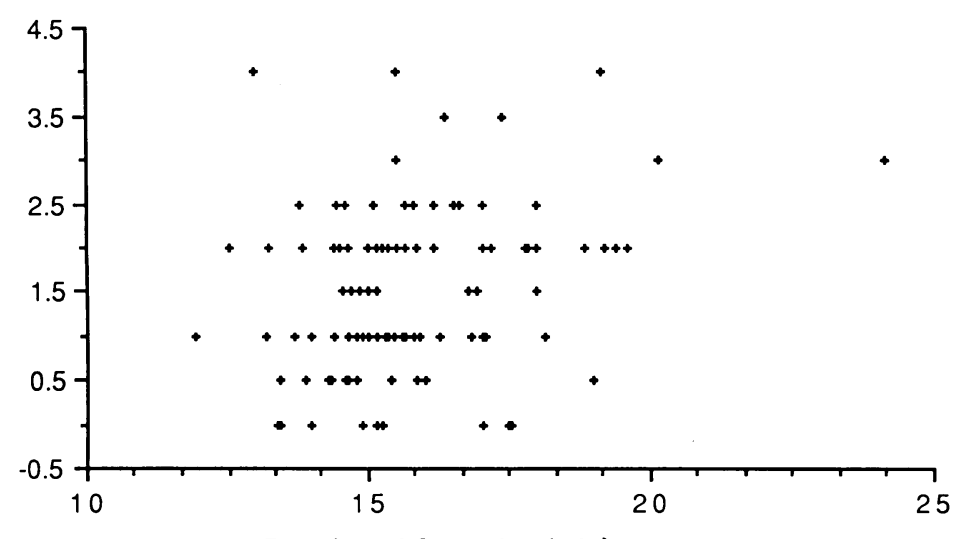

Duration of Operation (min)
TABLE I Initial and changes in pupil diameter by treatment and anaesthetic groups

\begin{tabular}{lll}
\hline & \multicolumn{2}{c}{ Pupil diameters in mm: mean $(S D)$, number } \\
\cline { 2 - 3 } & Treatment & Non-treatment \\
\hline Initial pupil diameter & $8 \cdot 1(1 \cdot 1) \mathrm{n}=63$ & $8 \cdot 3(1 \cdot 0) \mathrm{n}=50$ \\
Difference from preoperative pupil diameter \\
LA+GA & $1 \cdot 2(0 \cdot 9) \mathrm{n}=49$ & $1 \cdot 8(0 \cdot 8) \mathrm{n}=34$ \\
LA alone & $1 \cdot 3(1 \cdot 0) \mathrm{n}=14$ & $1 \cdot 9(1 \cdot 2) \mathrm{n}=16$ \\
Total & $1 \cdot 2(0 \cdot 9) \mathrm{n}=63$ & $1 \cdot 8(0 \cdot 9) \mathrm{n}=50$ \\
\multicolumn{3}{c}{} \\
Proportionate change from preoperative pupil diameter \\
LA+GA & $0 \cdot 15(0 \cdot 11) \mathrm{n}=49$ & $0 \cdot 21(0 \cdot 09) \mathrm{n}=34$ \\
LA alone & $0 \cdot 17(0 \cdot 13) \mathrm{n}=14$ & $0 \cdot 25(0 \cdot 17) \mathrm{n}=16$ \\
Total & $0 \cdot 16(0 \cdot 11) \mathrm{n}=63$ & $0 \cdot 22(0 \cdot 12) \mathrm{n}=50$ \\
\hline
\end{tabular}

pendently significant. The non-administration of indomethacin resulted in a larger perioperative reduction in pupil diameter. The age of the patient and the type of anaesthetic used were not significantly related to the reduction in pupil size. The mean reductions in pupil diameter in the two treatment groups are shown in Table I. Also in Table I are the mean changes in pupil diameters for each of the treatment groups subdivided by each of the anaesthetic groups. The treatment effect was the same for each of the anaesthetic groups. The numbers of eyes in each of the treatment and non-treatment groups that had at least $2.0 \mathrm{~mm}$ perioperative miosis were 20 $(31 \cdot 7 \%)$ and $30(60.0 \%)$ respectively. The operating times for the two groups were identical and were $15 \cdot 6$, SD $1.9(n=63)$ and $15 \cdot 6$, SD 1.9 $(n=50)$ minutes respectively.

\section{Discussion}

For efficient cataract surgery maintained mydriasis is necessary. We have shown that for one surgeon increased change in pupil diameter is associated with longer surgical procedures. By using topical aqueous indomethacin the amount of surgical miosis was reduced. Less than a $1 / 3$ $(31 \cdot 7 \%)$ of eyes underwent more than $2.0 \mathrm{~mm}$ of miosis compared with almost $2 / 3$ in the nontreatment group $(60.0 \%)$. This prevention of miosis was found in eyes of patients undergoing LA alone and LA plus GA. However, overall initial pupil diameters were greater for the LA and GA group. The anaesthetists in this unit did not use any premedication in patients over the age of 65 (25/113 eyes) in this study and did not use atropine as a premedication in the patients under the age of 65 . Droperidol, which was used in some of the younger patients as a premedication, may have a mild atropine effect. ${ }^{4}$ Apart from this tentative suggestion, the significant difference in the initial pupil size between the use of combined general and local anaesthesia and local anaesthesia alone in this centre is unexplained.

In view of the results of this study it is suggested that the minimum aqueous level of idomethacin needed to prevent surgical miosis may be obtained by administration less than 24 hours preoperatively. The use of topical indomethacin one hour preoperatively reduced surgical miosis and may be of practical benefit in both day case and inpatient cataract surgery.

We thank Ann Badger, secretary to Mr Pearce, for administrative help; Allan Davies, Assistant Regional Information Manager, West Midlands Regional Health Authority, and Lucy Titcomb, 
pharmacist in charge, the Birmingham and Midland Eye Hospital, for pharmacological advice.

1 Keates RH, McGowan KA. The effect of topical indomethacin ophthalmic solution in maintaining mydriasis during cataract ophthalmic solution in maintaining mydriasis
surgery. Ann Ophthalmol 1984; 16:1116-21.

2 Keulen-De-Vos HC, Van-Ri JG, Renardel-De-Lavalette JC,
Jansen JT. The effect of indomethacin in preventing surgically induced miosis. Br f Ophthalmol 1983; 67:94-6.

3 Rosner B. Mulivariate methods in ophthalmology with applications to other paired data situations. Biometrics 1984; 40: 102535.

4 Parmentier P, Dagnellie P. Has droperidol an atropinic effect? BrF Anaesth 1979; 51 : 775. 